\title{
Stability and Bifurcation: Discrete Differential Algebraic Planar Model with Square Root Response
}

\author{
A. George Maria Selvam, R. Janagaraj, Ozlem Ak Gumus.
}

\begin{abstract}
The stability and existence of bifurcation analysis for a two spices discrete time model by introducing square root functional response and step size is examined in this work. Forward Euler scheme method is applied to formulate the discrete model from the continuous model, particularly to explore the rich dynamical behavior of the proposed model. Because the model has square root response function, trivial and axial equilibrium positions are singular. In order to discuss the stability of the trivial and axial equilibrium positions, a transformation is applied. Moreover, we explore the stability of the interior equilibrium position in a discrete two spices model using jury conditions. The numerical experiments are performed for distinct parameter values and also time series and phase line diagrams are presented. We also apply bifurcation theory to find whether the model of spices undergoes periodic doubling bifurcation at its axial and interior equilibrium positions. Numerical examples are provided and they exhibit rich dynamics in both species, including, period $-2,4,8 \& 16$ orbits, periodic windows and Non periodic orbit(chaos).
\end{abstract}

Keywords: Discrete Time, Equilibrium Positions, Stability, Periodic - Doubling Bifurcations.

\section{INTRODUCTION}

Over the past few centuries the explosion in scientific knowledge and technology has been a contributing factor and also to some extent a direct consequence in advancement of mathematical modeling. The development of more contemporary form of mathematical modeling, has further enhanced our understanding of the world around us. A periodic motivation for those who create or develop mathematical models is the desire to form prediction. [4] Mathematical model necessitates a system of equations or an equation, which explains some physical problem or phenomenon that has its origin in economics, science and technology, engineering, or some other fields. The subject of mathematical modeling involves physical observation, formation of equations from physical to mathematical, solution methods, and mathematical analysis.

During the past centuries, many types of models have been developed to investigate the natural and social processes that enlarge over time. Now-a-days, these models are referred as dynamical systems. [7] Dynamical systems are divided into two general categories, i.e. deterministic models and stochastic models respectively. Deterministic models are

Revised Manuscript Received on December 5, 2019.

George Maria Selvam A., Department of Mathematics, Sacred Heart College (Autonomous), Tirupattur-635601, Tamil Nadu, India

Email : agmshc@gmail.com

Janagaraj R., Department of Mathematics, Sacred Heart College (Autonomous), Tirupattur-635601, Tamil Nadu, India

Ozlem Ak Gumus, Department of Mathematics, Faculty of Arts and Sciences, Adiyaman University, Adiyaman - 02040, Turkey ordinarily employed when the number of quantities involved in the process being modeled is relatively small and all the underlying scientific principles are fairly well understood.

The rest of paper is organised as follows: Section 2 describes both Lotka - Volterra continuous and discrete time dynamical systems. We establish the existence of equilibrium positions and variation matrix of the modified two species system of square root response function with step size in section 3. Section 4 analyzes the stability of the trivial and axial equilibrium positions for the system. In Section 5, we investigate the stability of interior positive equilibrium position for the system and the numerical example with simulation is presented. In Section 6, we identify the existence of the periodic doubling bifurcation for both axial and interior equilibrium positions and provide bifurcation diagrams of distinct periodic orbits for suitable parameter values. Also illustration for sensitive analysis of initial conditions is presented. Finally the work ends with a brief conclusion in the last section.

\section{BASIC LOTKA - VOLTERRA TWO SPICES MODEL}

Ecology is that branch of biology dealing with understanding of how species relate with each other and their environment essentially the inter and intra relationships of the species. In particular, it encompasses aspects such as population growth, competition, symbiotic ecologic relationships (mutualism), trophic relations, migration and physical environment interactions. Such population dynamics have enthused biologist and mathematicians alike who collectively enhanced the study of interactions of species by developing population based mathematical models.

In mathematical ecology, prey-predator interaction plays a fundamental role due to its importance and universal existence. The dynamical relationship between predator and prey populations, which is one of the vital themes in mathematical ecology is helpful in the preservation of speices in a habitat.

Verhulst was foremost to introduce the continuous time basic population model to study the growth rate of single species in population. At the onset of $19^{\text {th }}$ century Lotka and Volterra pioneered the study of predator-prey interaction. It is the one of the most fundamental and simplest interaction population models in ecology. In any biological system, it is the first strategic attempt to develop a model based on the quantitative observations. 
In ecology, several authors discussed the dynamics of predator-prey model and contributed to the development of the population dynamics $[2,6,13]$.

\section{A.Continuous Time Two Spices Model}

The dynamical nature of the predator-prey model is one of the most interesting subjects in ecology. Also this is defined by dynamical models or differential equations. Quite a few authors have studied the square root model [15, 9]. Braza [3] discussed the prey-predator model of square root response function with time delay. Let us consider the following model of two species square root response function:

$$
\begin{aligned}
& \dot{x}=\mu x(l)-\mu x^{2}(l)-\alpha \sqrt{x(l) y(l)} \\
& \dot{y}=-\beta y(l)+\gamma \sqrt{x(l) y(l)}
\end{aligned}
$$

where $\mu, \alpha, \beta, \gamma$ are all positive.

\section{B.Discrete Model Of Square Root Function With Step Size}

In recent years, $[1,5,8,10,11]$ due to the following three reasons the discrete-time predator prey models have come to the fore front: First one is, in comparision to continuous time models, the discrete-time models are more suitable to describe the species with non overlapping generations and the second one is discrete models exhibits more complex and rich dynamics. Finally, we get accurate numerical solutions from discrete-time models.

By applying the Euler scheme to the square root response function model (1), we get the modified discrete differential algebraic planar model with square root response and step size as follows:

$$
\begin{aligned}
& x(l+1)=x(l)+\delta\left[\mu x(l)-\mu x^{2}(t)-\alpha \sqrt{x(l) y(l)}\right] \\
& y(l+1)=y(l)+\delta[\gamma \sqrt{x(l) y(l)}-\beta y(l)]
\end{aligned}
$$

where $x(l)$ and $y(l)$ denote the population density of prey and predator at time $l$ respectively, $\delta>0$ is the step size, $\mu>0$ denotes the intrinsic growth rate of prey, $\alpha>0$ is the coefficient of predation, $\gamma>0$ represents the coefficient of conversion and $\beta>0$ is the natural death rate of the predator species.

\section{III.EQUILIBRIUM POSITIONS AND VARIATIONAL MATRIX OF MODEL (2)}

We discover the equilibrium positions of the model (2) by calculating the variational matrix of the predator-prey model (2). To determine the equilibrium positions we need to solve the following nonlinear system:

$$
\begin{aligned}
& x=x+\delta\left[\mu x-\mu x^{2}-\alpha \sqrt{x y}\right] \\
& y=y+\delta[\gamma \sqrt{x y}-\beta y]
\end{aligned}
$$

By solving the equation (3), we obtain three non-negative equilibrium positions,

- $\mathrm{EP}_{0}=(0,0)$ is trivial position.

- $\mathrm{EP}_{1}=(1,0)$ is axial equilibrium position.
- $\mathrm{EP}_{2}=\left(x^{*}, \frac{\gamma^{2}}{\beta^{2}} x^{*}\right)$ is the interior positive equilibrium position which is feasible only if $\mu>\frac{\gamma \alpha}{\beta}$, where $x^{*}=1-\frac{\gamma \alpha}{\mu \beta}$.

Now, we examine the behavior of the model (2) around each of the above equilibrium positions. The variation matrix of model (2) at the position variable $(x, y)$ is

$V(x, y)=\left[\begin{array}{cc}1+\delta\left[(1-2 x) \mu-\frac{\alpha}{2} \sqrt{\frac{y}{x}}\right] & -\frac{\alpha \delta}{2} \sqrt{\frac{x}{y}} \\ \frac{\delta \gamma}{2} \sqrt{\frac{y}{x}} & 1+\delta\left[\frac{\gamma}{2} \sqrt{\frac{x}{y}}-\beta\right]\end{array}\right]$

The characteristic equation of (2) is

$F(m)=m^{2}-T m+D=0$

where $\quad T=\delta\left[(1-2 x) \mu-\beta-\frac{1}{2}\left[\alpha \sqrt{\frac{y}{x}}-\gamma \sqrt{\frac{x}{y}}\right]\right]+2 \quad$ and $D=(1-\delta \beta)\left(1+\delta\left[(1-2 x) \mu-\frac{\alpha}{2} \sqrt{\frac{y}{x}}\right]\right)+(1+\delta \mu(1-2 x)) \frac{\delta \gamma}{2} \sqrt{\frac{x}{y}}$, are the trace and determinant of the matrix $V(x, y)$ respectively.

Following lemma aids to analyze the stability of the equilibrium positions of the model, which is estimated by the relations between roots and coefficients of a quadratic equation. [12, 14, 16]

Lemma 1: Consider the characteristic equation (5) and $\xi_{1}, \xi_{2}$ be the roots of $\Lambda(\xi)=0$. Suppose that $\Lambda(1)>0$. Then we have

- $\left|\xi_{1}\right|<1$ and $\left|\xi_{2}\right|<1 \Leftrightarrow \Lambda(-1)>0 \& \Lambda(0)<1$.

- $\left|\xi_{1}\right|<1$ and $\left|\xi_{2}\right|>1$ (or $\left|\xi_{1}\right|>1$ and $\left|\xi_{2}\right|<1$ ) $\Leftrightarrow \Lambda(-1)<0$.

- $\left|\xi_{1}\right|>1$ and $\left|\xi_{2}\right|>1 \Leftrightarrow \Lambda(-1)>0 \& \Lambda(0)>1$.

- $\left|\xi_{1}\right|=-1$ and $\left|\xi_{2}\right| \neq 1 \Leftrightarrow \Lambda(-1)=0 \& \mathrm{~T} \neq 0 \& 2$.

- $\xi_{1}$ and $\xi_{2}$ are complex and $\left|\xi_{1}\right|=\left|\xi_{2}\right| \Leftrightarrow$ $\mathrm{T}^{2}-4 \mathrm{D}<0 \quad \& \Lambda(0)=1$.

\section{IV.STABILITY EXPLORATION OF TRIVIAL AND AXIAL EQUILIBRIA}

The stability analysis of trivial and axial equilibrium positions are investigated in this section. Here the model has the square root response function term, so that the trivial point and axial euilibrium positions are singular. It is obvious that the variation matrix (3) is in-determinant at $\mathrm{EP}_{0}$ and $\mathrm{EP}_{1}$. In order to clearly discuss the stability of the trivial and axial equilibrium points, we rearrange the variables $x(l)$ and $y(l)$ by $X^{2}(l)$ and $Y^{2}(l)$ respectively; so model (2) can be re written in the following form: 


$$
\begin{aligned}
& X(l+1)=X(l)+\delta\left[\frac{\mu}{2} X(l)-\frac{\mu}{2} X^{3}(l)-\frac{\alpha}{2} Y(l)\right] \\
& Y(l+1)=Y(l)+\delta\left[\frac{\gamma}{2} X(l)-\frac{\beta}{2} Y(l)\right]
\end{aligned}
$$

With the help of variational matrix of model (6) and applying the trivial and axial equilibrium positions, we have the following results.

Proposition 1: The trivial equilibrium positions $\mathrm{EP}_{0}$ is

- $\operatorname{sink}$ if $\mu \in\left(\frac{\delta(\alpha \gamma \delta-4 \beta)+16}{\delta(\delta \beta-4)}, \frac{2 \beta-\delta \alpha \gamma}{2-\delta \beta}\right)$,

- source if $\mu>\max \left\{\frac{\delta(\alpha \gamma \delta-4 \beta)+16}{\delta(\delta \beta-4)}, \frac{2 \beta-\delta \alpha \gamma}{2-\delta \beta}\right\}$.

Proof. The variation matrix at trivial position $\mathrm{EP}_{0}$ is as follows:

$V\left(\mathrm{EP}_{0}\right)=\left[\begin{array}{cc}1+\frac{\delta \mu}{2} & -\frac{\delta \alpha}{2} \\ \frac{\delta \gamma}{2} & 1-\frac{\delta \beta}{2}\end{array}\right]$.

The characteristic equation of $V\left(\mathrm{EP}_{0}\right)$ satisfies $F(m)=m^{2}-T m+D=0$, where $T=2+\frac{\delta}{2}(\mu-\beta)$ and $D=1+\frac{\delta}{2}(\mu-\beta)-\frac{\delta^{2}}{4}(\mu \beta-\alpha \gamma)$. Also the eigen values of the variation matrix $V\left(\mathrm{EP}_{0}\right)$ are $m_{1,2}=1+\frac{\delta}{4}\left[(\mu-\beta) \pm \sqrt{(\mu-\beta)^{2}-4 \alpha \gamma}\right]$. Using Jury's criterion, it is a sink if $\mu \in\left(\frac{\delta(\alpha \gamma \delta-4 \beta)+16}{\delta(\delta \beta-4)}, \frac{2 \beta-\delta \alpha \gamma}{2-\delta \beta}\right)$ and source if $\mu>\max \left\{\frac{\delta(\alpha \gamma \delta-4 \beta)+16}{\delta(\delta \beta-4)}, \frac{2 \beta-\delta \alpha \gamma}{2-\delta \beta}\right\}$.

Corollary: The trivial position $\mathrm{EP}_{0}$ is a saddle if $\mu<\frac{\delta(\alpha \gamma \delta-4 \beta)+16}{\delta(\delta \beta-4)}$ and Non-hyperbolic if either $\mu=\frac{\delta(\alpha \gamma \delta-4 \beta)+16}{\delta(\delta \beta-4)}$ or $\mu=\frac{2 \beta-\delta \alpha \gamma}{2-\delta \beta}$.

Proposition 2: The axial equilibrium position $\mathrm{EP}_{1}$ is

- $\operatorname{sink}$ if $\mu \in\left(\frac{\delta(\delta \alpha \gamma-4 \beta)+16}{2 \delta(4-\delta \beta)}, \frac{2 \beta-\delta \alpha \gamma}{2(\delta \beta-2)}\right)$,

- source if $\mu>\max \left\{\frac{\delta(\delta \alpha \gamma-4 \beta)+16}{2 \delta(4-\delta \beta)}, \frac{2 \beta-\delta \alpha \gamma}{2(\delta \beta-2)}\right\}$.

Proof. Applying the axial equilibrium position $\mathrm{EP}_{1}$ to the variation matrix we have

$$
V\left(\mathrm{EP}_{1}\right)=\left[\begin{array}{cc}
1-\delta \mu & -\delta \frac{\alpha}{2} \\
\delta \frac{\gamma}{2} & 1-\delta \frac{\beta}{2}
\end{array}\right]
$$

Solving the matrix $V\left(\mathrm{EP}_{1}\right)$ and get the characteristic equation

$$
F(m)=m^{2}-T m+D=0
$$

where
$T=2-\delta\left[\mu+\frac{\beta}{2}\right]$ and $D=1-\left(\mu+\frac{\beta}{2}\right) \delta+\frac{\delta^{2}}{2}\left(\mu \beta+\frac{\alpha \gamma}{2}\right)$.

Also $m_{1,2}=1-\frac{\delta}{2}\left[\left(\mu+\frac{\beta}{2}\right) \pm \sqrt{\left(\mu-\frac{\beta}{2}\right)^{2}-\alpha \gamma}\right]$ are eigen values of $V\left(\mathrm{EP}_{1}\right)$. Using Jury's criterion, it is a sink if $\mu \in\left(\frac{\delta(\delta \alpha \gamma-4 \beta)+16}{2 \delta(4-\delta \beta)}, \frac{2 \beta-\delta \alpha \gamma}{2(\delta \beta-2)}\right)$ and source if $\mu>\max \left\{\frac{\delta(\delta \alpha \gamma-4 \beta)+16}{2 \delta(4-\delta \beta)}, \frac{2 \beta-\delta \alpha \gamma}{2(\delta \beta-2)}\right\}$. This completes the proof.

Corollary: The axial equilibrium position $E P_{1}$ is a saddle if $\mu<\frac{\delta(\delta \alpha \gamma-4 \beta)+16}{2 \delta(4-\delta \beta)}$ and Non-hyperbolic if either $\mu=\frac{\delta(\delta \alpha \gamma-4 \beta)+16}{2 \delta(4-\delta \beta)}$ or $\mu=\frac{2 \beta-\delta \alpha \gamma}{2(\delta \beta-2)}$.

\section{DYNAMICS OF STABILITY IN POSITIVE EQUILIBRIUM POSITION OF (2) AND ITS NUMERICAL SIMULATIONS}

We investigate the stability of positive equilibrium position $\mathrm{EP}_{2}$ by finding the variational matrix

$V\left(\mathrm{EP}_{2}\right)=\left[\begin{array}{cc}1+\delta\left[\frac{3 \alpha \gamma}{2 \beta}-\mu\right] & -\frac{\delta \alpha \beta}{2 \gamma} \\ \frac{\delta \gamma^{2}}{2 \beta} & 1-\frac{\delta \beta}{2}\end{array}\right]$.

The characteristic polynomial is $F(m)=m^{2}-T m+D=0$, where

$$
T=2+\delta\left[\frac{3 \alpha \gamma}{2 \beta}-\mu-\frac{\beta}{2}\right]
$$

and $D=1+\delta\left[\frac{3 \alpha \gamma}{2 \beta}-\mu-\frac{\beta}{2}\right]+\frac{\delta^{2}}{2}[\mu \beta-\alpha \gamma]$. Then the eigen values are

$m_{1,2}=1+\frac{\delta}{2}\left[\left(\frac{3 \alpha \gamma}{2 \beta}-\mu-\frac{\beta}{2}\right) \pm \sqrt{\left(\frac{3 \alpha \gamma}{2 \beta}-\mu-\frac{\beta}{2}\right)^{2}-2(\mu \beta-\alpha \gamma)}\right]$.

Considering Jury's principle, we have

I. (a) $S^{*} \geq 0$ and $\mu>\frac{2}{\delta}+\frac{\alpha \gamma}{\beta}\left[1-\frac{2}{\delta \beta-4}\right]$,

(b) $S^{*}<0$ and $\mu<\frac{\beta}{\delta \beta-2}+\frac{\alpha \gamma}{\beta}\left[1-\frac{1}{\delta \beta-2}\right]$.

II. (a) $S^{*} \geq 0$ and $\mu>\frac{2}{\delta}+\frac{\alpha \gamma}{\beta}\left[1-\frac{2}{\delta \beta-4}\right]$,

(b) $S^{*}<0$ and $\mu>\frac{\beta}{\delta \beta-2}+\frac{\alpha \gamma}{\beta}\left[1-\frac{1}{\delta \beta-2}\right]$. 
III. (a) $S^{*} \geq 0$ and $\mu<\frac{2}{\delta}+\frac{\alpha \gamma}{\beta}\left[1-\frac{2}{\delta \beta-4}\right]$,

(b) $S^{*} \geq 0$ and $\mu>\frac{2}{\delta}+\frac{\alpha \gamma}{\beta}\left[1-\frac{2}{\delta \beta-4}\right]$.

IV. (a) $S^{*} \geq 0$ and $\mu=\frac{2}{\delta}+\frac{\alpha \gamma}{\beta}\left[1-\frac{2}{\delta \beta-4}\right]$,

(b) $S^{*}<0$ and $\mu=\frac{\beta}{\delta \beta-2}+\frac{\alpha \gamma}{\beta}\left[1-\frac{1}{\delta \beta-2}\right]$.

Here $S^{*}=\delta^{2}\left[\left(\frac{3 \alpha \gamma}{2 \beta}-\mu-\frac{\beta}{2}\right)^{2}-2(\mu \beta-\alpha \gamma)\right]$.

Proposition 3: Consider $\mu>\frac{\alpha \gamma}{\beta}$.

- If the condition (I) holds, then $\mathrm{EP}_{2}$ is stable (sink).

- If the condition (II) holds, then $\mathrm{EP}_{2}$ is unstable (source).

- If the condition (III) holds, then $\mathrm{EP}_{2}$ is saddle.

- If the condition (IV) holds, then $\mathrm{EP}_{2}$ is non-hyperbolic.

Now, we present some arithmetical indication for the qualitative dynamical nature of the model (2). For example, consider the parameter values

$\delta=0.85 ; \mu=2.8 ; \alpha=1.5 ; \beta=1.999 ; \gamma=2.7$, the model (2) can be rewritten as

$$
\begin{aligned}
& x(\ell+1)=x(\ell)+0.85\left[2.8 x(\ell)-2.8 x^{2}(\ell)-1.5 \sqrt{x(\ell) y(\ell)}\right] \\
& y(\ell+1)=y(\ell)+0.85[2.7 \sqrt{x(\ell) y(\ell)}-1.999 y(\ell)]
\end{aligned}
$$

The interior equilibrium position is $\mathrm{EP}_{2}=(0.286,0.504) \&$ the variational matrix of the above model is $V(0.286,0.504)=\left[\begin{array}{cc}1.2032 & -0.4720 \\ 1.5499 & 0.1504\end{array}\right]$. Here $T=1.3536$, $D=0.9125$ and $F(-1)=3.2661>0, F(0)=0.9125<1$. Also the eigen values of $\mathrm{EP}_{2}$ are $\left|m_{1,2}\right|=0.9553<1$. From Proposition 3, we can easily see that the model is stable. Also from Figure - 1, the phase portrait is starting at the initial condition $(0.55,0.45)$ which spirals inwards to $(0.286,0.504)$, indicating stability.

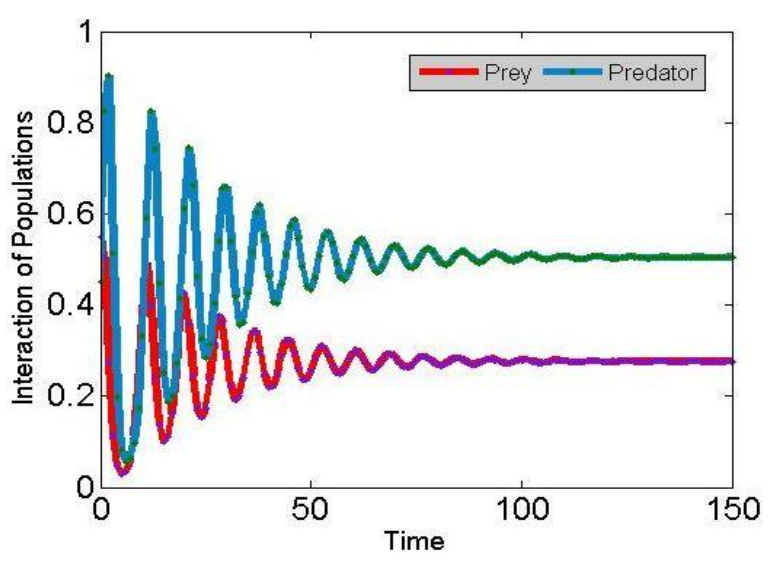

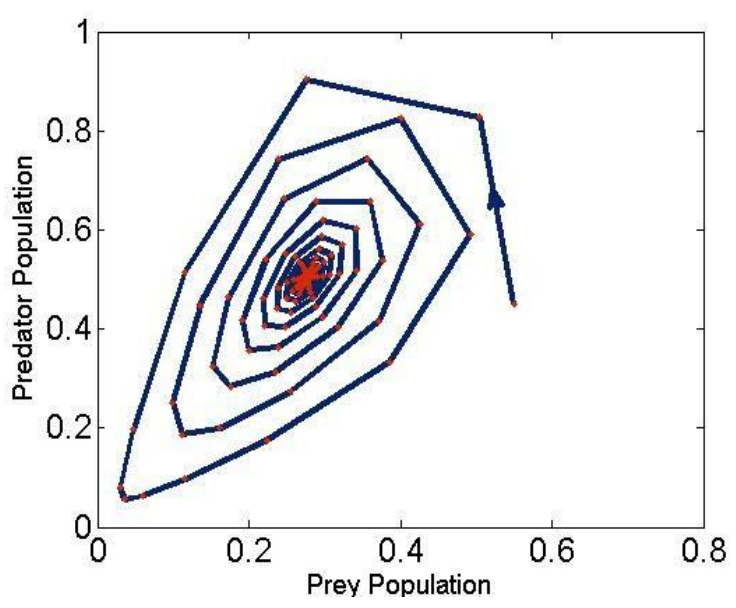

Fig. 1. Dynamics of Stability at Interior Equilibrium Position of the System (2)

\section{VI.ANALYSIS OF PERIODIC-DOUBLING BIFURCATIONS}

In this section, bifurcation theory is applied in order to examine the existence of period-doubling (flip) bifurcation at axial equilibrium position $\mathrm{EP}_{1}$ for the system (6) and positive equilibrium position $\mathrm{EP}_{2}$ of the model (2). Let $\mu$ be the bifurcation parameter and taking $\mu \equiv \mu_{0}=\frac{\delta(\delta \alpha \gamma-4 \beta)+16}{2 \delta(4-\delta \beta)}$.

At $\mu=\mu_{0}$, the characteristic equation is

$$
F(\lambda)=(\lambda+1)\left(\lambda-1+\frac{\delta^{2} \alpha \gamma}{2(4-\delta \beta)}+\frac{\delta \beta}{2}\right) \text {. }
$$

Now, we have

$\lambda_{2}=1-\frac{\delta^{2} \alpha \gamma}{2(4-\delta \beta)}-\frac{\delta \beta}{2}$.

(9)

Then, the roots of equation (8) are $\lambda_{1}=-1$ and $\lambda_{2}$ is defined in equation (9). Moreover, consider the following set, $\mathrm{EP}_{1}(\mathrm{PD})=\left\{\begin{array}{l}\left(\delta, \alpha, \beta, \gamma, \mu_{0}\right): \delta>0, \alpha>0, \beta>0, \gamma>0, \\ \mu_{0}=\frac{\delta(\delta \alpha \gamma-4 \beta)+16}{2 \delta(4-\delta \beta)},\left|\lambda_{2}\right| \neq \pm 1\end{array}\right\}$.

Also we can see that the existence of periodic doubling bifurcation for the model (2), we fix $\mu$ as bifurcation parameter and choosing $\mu \equiv \mu_{0}=\frac{2}{\delta}+\frac{\alpha \gamma}{\beta}\left[1-\frac{2}{\delta \beta-4}\right]$. At $\mu=\mu_{0}$, the characteristic equation is

$F(\lambda)=(\lambda+1)\left(\lambda-1-\frac{\delta \alpha \gamma}{2 \beta}-\frac{2 \delta \alpha \gamma}{\beta(\delta \beta-4)}+\frac{\delta \beta}{2}\right)$.

Now, we have

$\lambda_{2}=1+\frac{\delta \alpha \gamma}{2 \beta}+\frac{2 \delta \alpha \gamma}{\beta(\delta \beta-4)}-\frac{\delta \beta}{2}$.

Then, $\lambda_{1}=-1$ and $\lambda_{2}$ is defined in equation (11) are the roots of the equation (10). In-addition, consider the following set, 
$\mathrm{EP}_{2}(\mathrm{PD})=\left\{\begin{array}{l}\left(\delta, \alpha, \beta, \gamma, \mu_{0}\right): \delta>0, \alpha>0, \beta>0, \gamma>0, \\ \mu_{0}=\frac{2}{\delta}+\frac{\alpha \gamma}{\beta}\left[1-\frac{2}{\delta \beta-4}\right],\left|\lambda_{2}\right| \neq \pm 1\end{array}\right\}$.

Figure $-2(A) \& 2(B)$ show the periodic doubling bifurcation diagrams in $(\mu-x)$ and $(\mu-y)$ planes as $\mu$ varys and fixing the

parameters

$\delta=0.85, \alpha=1.05, \beta=1.999, \gamma=1.467$ respevtively. It is clear that the orbit with initial point $(0.55,0.45)$ moves to the stable equilibrium position $\mathrm{EP}_{2}$ for $\mu<3.7933$ and a discrete periodic doubling bifurcation occurs at $\mu=3.7933$. Also quasi periodic orbits on the invariant cycle arise for $\mu>3.7933$ and periodic orbits emerge in the periodic window, the orbits tend to chaos with the increases of $\mu$.

Numerically, to the characteristic equation (8) by applying the above parameter values, we get $\lambda_{1}=-1$ and $\lambda_{2}=-0.0914$, which satisfies the periodic doubling bifurcation condition. Figure - 2(C) \& 2(D) are local amplifications of Figure - 2(A) \& 2(B), exhibiting periodic window occuring in the chaotic region. In periodic window, see Figure: 3(D-F), emerges period -8 orbits. In addition, each window is split in to a sub periodic doubling cascade and leads to chaos.
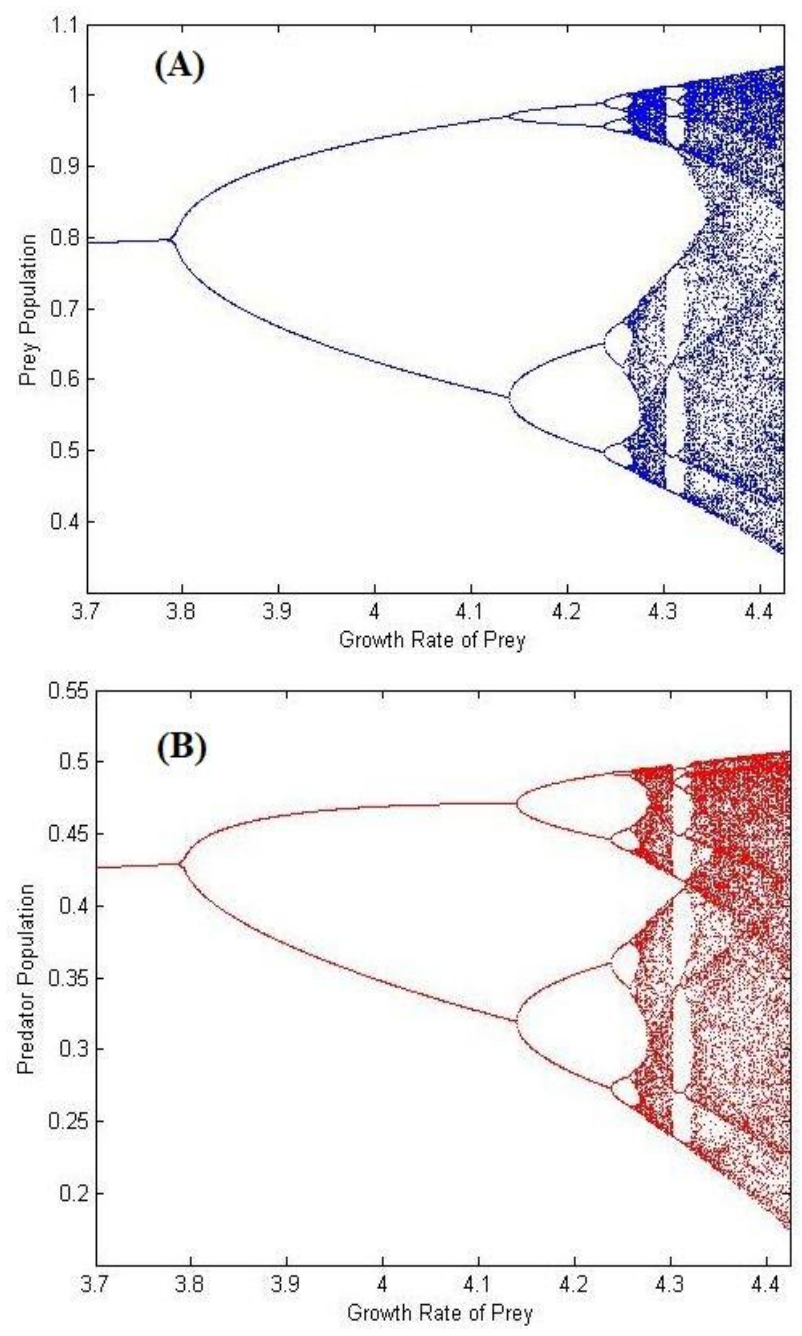

Retrieval Number: D10601284S519/2019@BEIESP
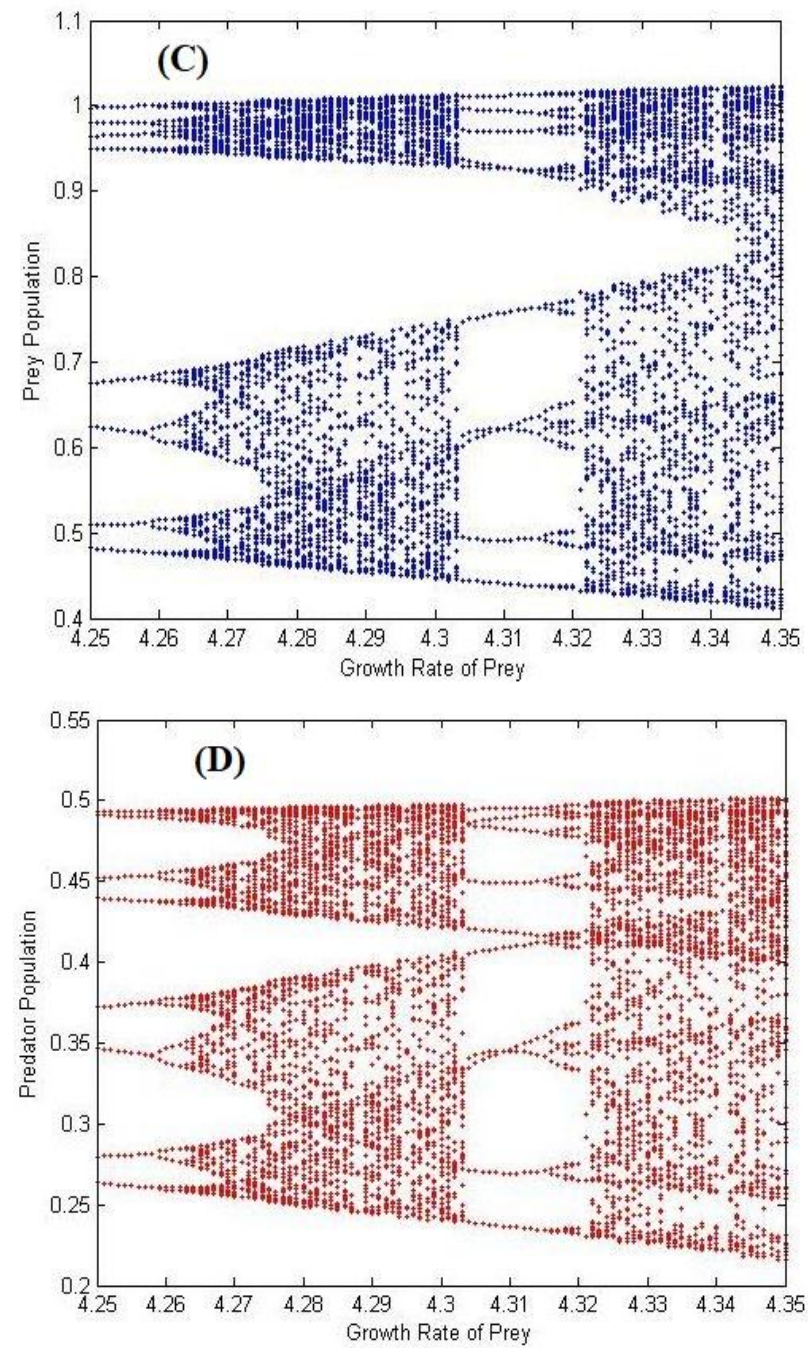

Fig. 2. Bifurcation Structure of (A) Prey Population; (B) Predator Population for the Model (2); (C \& D) Local Amplifications of (A) and (B)

Figure - 3(A) displays periodic doubling bifurcation diagram of the predator- prey model of (2) as $\mu$ varys in the interval $[2.2,3.5]$ and fixing the other parameter values as $\delta=0.85, \alpha=0.001, \beta=0.01, \gamma=0.1$. Under such conditions, we have $\lambda_{1}=-1$ and $\lambda_{2}=-0.0914$ at $\mu=2.3539$, which satisfies the periodic doubling bifurcation condition. Also, clearly the orbit with the initial points $(0.55,0.45)$ tends to the stable equilibrium position $\mathrm{EP}_{1}$ for $\mu<2.3539$, and a discrete periodic doubling bifurcation occurs at $\mu=2.3539$. As $\mu$ increases, the axial equilibrium position becomes unstable and periodic orbits emerge in the periodic window and the orbits approach to chaos. Figure - 3(C)-3(F) are local amplifications of Figure3(A), displaying periodic 8 orbits, periodic 16 orbits and periodic windows occuring in the chaotic region. In periodic windows, emerges periodic -6 orbits, periodic -5 orbits and periodic -3 orbits.

In addition, each window is split in to sub periodic doubling cascade leading to chaos see Figure-4. Moreover the coresponding Maximal Lyapunov Exponent (MLE) of model (2) displays in Figure-3(B). From Figure-3(B), the degree of the local stability for various 
values of $\mu \in(3,3.5)$ can easily be determined. MLE is positive at the values of $\mu>3$.
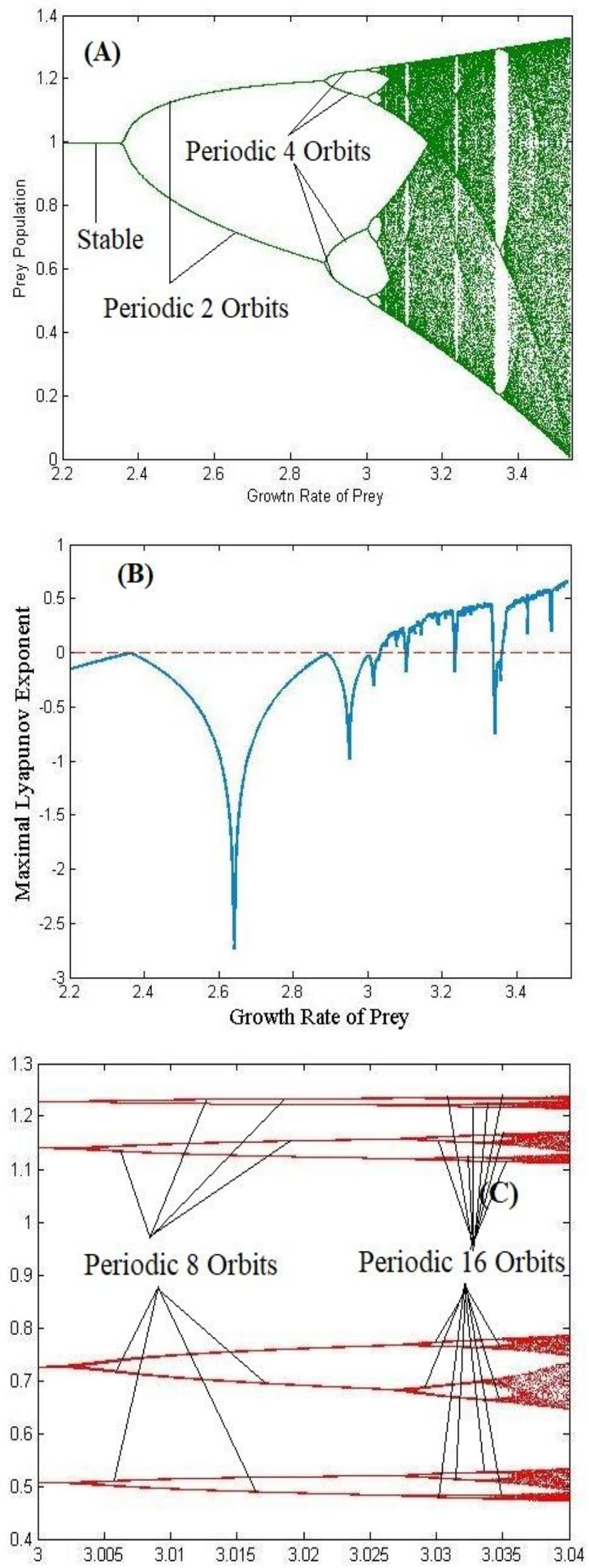
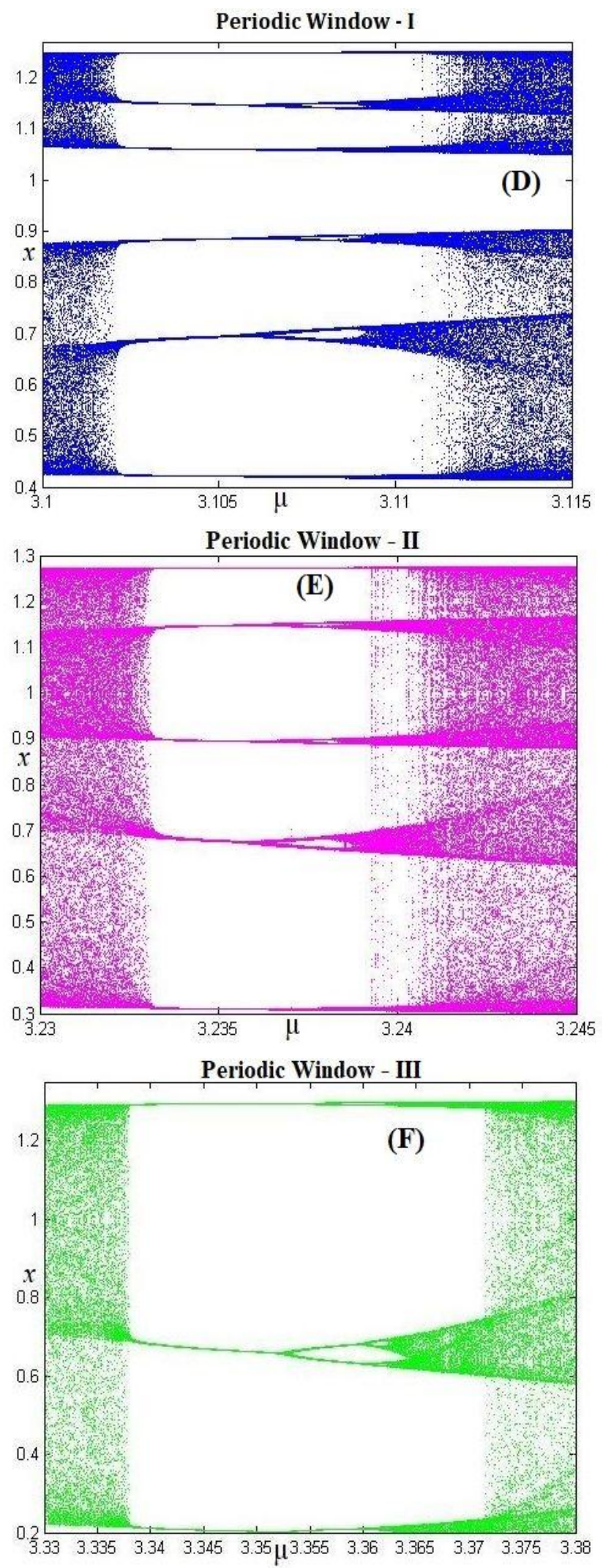

Fig. 3. (A) Bifurcation Diagram for the Prey Population of the Model (2); (B) Lyapunov exponent of the Model (2); (C-F) Local Amplifications of (A).

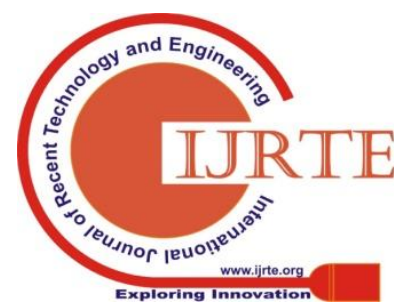



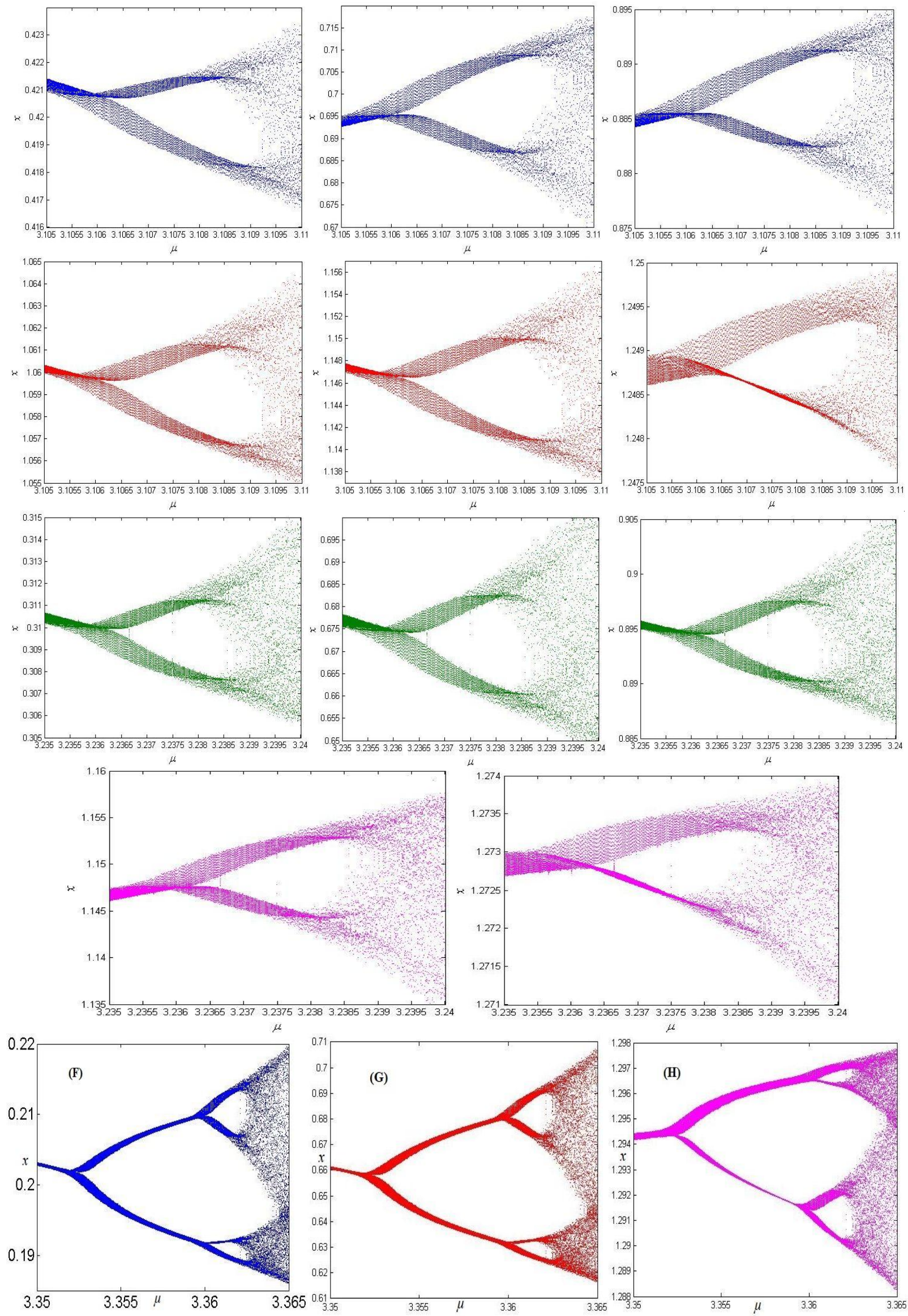

Fig. 4. A Sub Periodic Doubling Cascade Bifurcation of Periodic Windows I, II, \& III

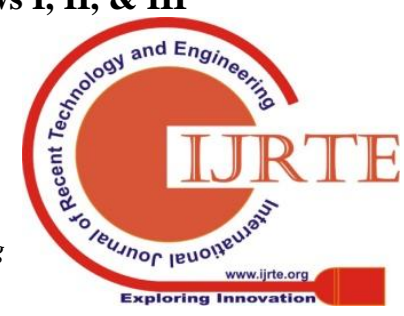




\section{A. Sensitive Analysis}

A characteristic behavior of chaos is the dependence on the sensitivity to initial points. To exhibit the sensitivity to initial values of model (2), two paths $\left(x_{0}+0.0001, y_{0}\right)$ and $\left(x_{0}, y_{0}+0.0001\right)$ are considered with initial points $\left(x_{0}, y_{0}\right)$ respectively. The results are established and presented in Figure-5 and Figure-6.
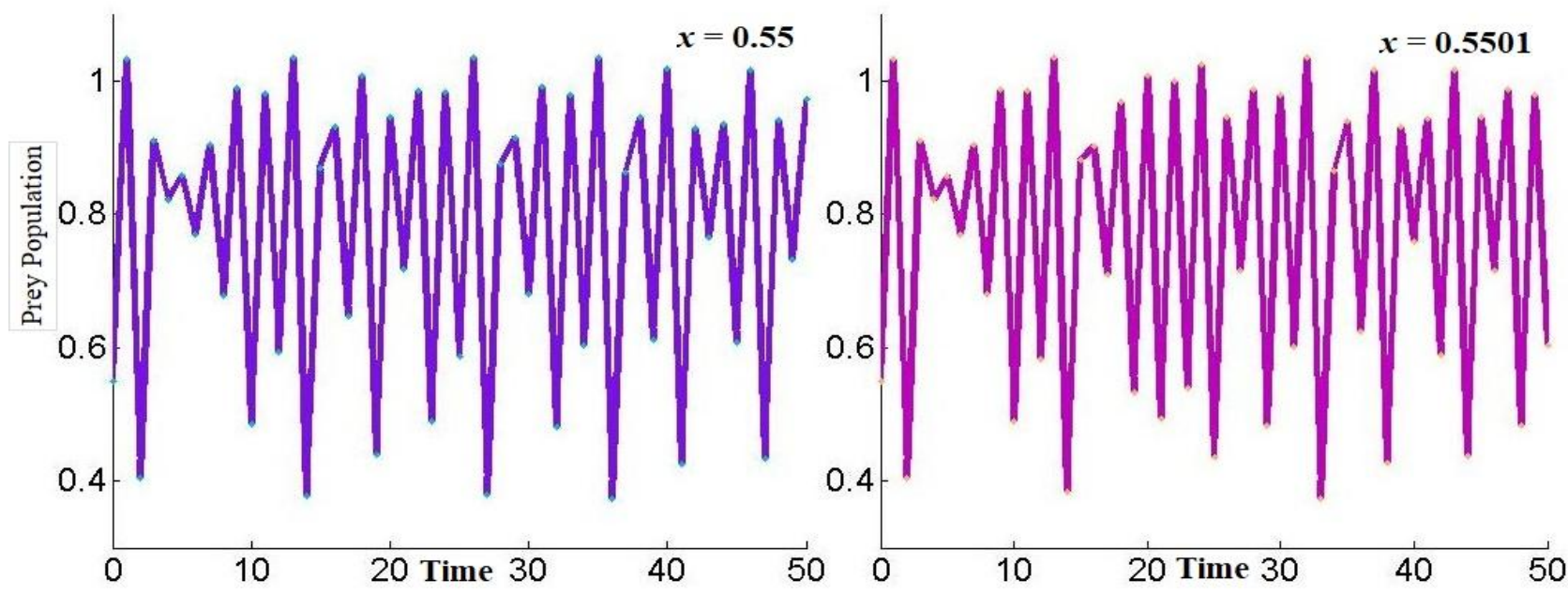

Fig. 5. Dynamics of the Sensitive for the Prey Population of (2)

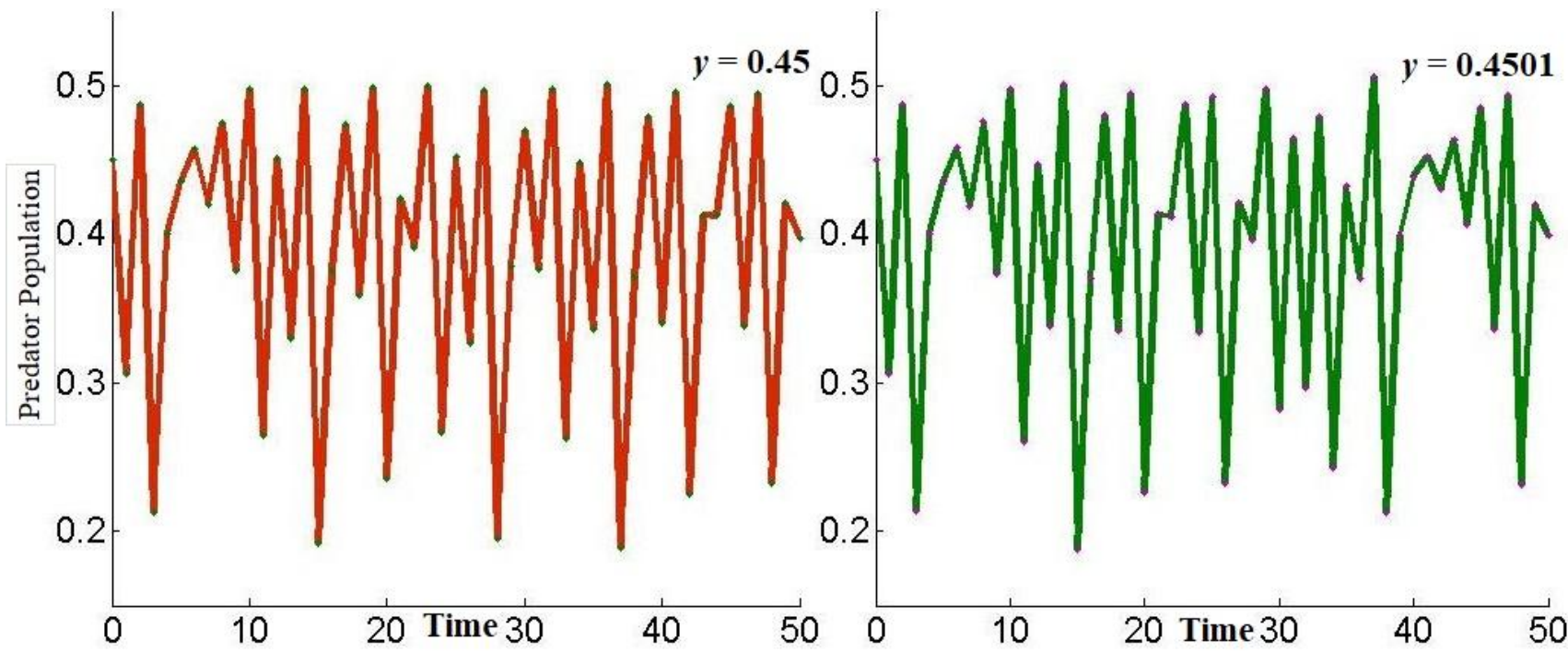

Fig. 6. Dynamics of the Sensitive for the Predator Population of (2)

\section{CONCLUSION}

In this work, we investigated the qualitative behavior of a modified prey predator discrete time model by introducing square root response function. Euler's scheme was implemented and the discrete model from the continuous model was obtained to study the rich dynamical behavior of the proposed model. The trivial and axial equilibrium positions were singularity because the model had square root response function. In order to analyze the stability of the trivial and axial equilibrium positions, we rescaled the variables as $x(l)=X^{2}(l)$ and $y(l)=Y^{2}(l)$. Moreover, we applied jury conditions to examine the stability of equilibrium positions in the discrete prey predator model. The numerical simulations are shown for distinct parameter values and plotted the time series diagrams with phase lines. Also we observed both equilibrium position of the model undergoes periodic doubling bifurcation with varrying $\mu$.
When $\mu$ increases there occurs periodic 2,4,8,16 and periodic windows which lead to chaos. Numerical examples justified that the model exhibits rich chaotic dynamics in both species.

\section{REFERENCES}

1. Abd-Elalim A. Elsadany, H. A. EL-Metwally, E. M. Elabbasy, H. N Agiza, "Chaos and bifurcation of a nonlinear discrete prey-predator system," Computational Ecology and Software, vol. 2, 2012, pp 169-180.

2. T. Agrawal, M. Saleem, "Complex dynamics in a ratio-dependent two-predator one-prey model," Comput. Appl. Math., vol 34, 2015, pp 265-274.

3. P. A. Braza, "Predator-prey dynamics with square root functional responses," Nonlinear Anal., vol 13, 2012, pp 1837-1843.

4. J. David Logan, "A First Course in Differential Equations," Second Edition, Springer, New York, 2011.

5. Q. Din, "Complexity and chaos control in a discrete-time prey predator model," Communications in Nonlinear Science and Numerical Simulation, vol. 49, 2017, pp. 113-134. 
6. J. Dhar, "A prey-predator model with diffusion and a supplementary resource for the prey in a two-patch environment," Math. Model. Anal., vol 9, 2004, pp 9-24.

7. Frederick R. Marotto, "Introduction to Mathematical Modeling Using Discrete Dynamical Systems," Thomson Brooks/Cole, USA, 2006.

8. Hunki Baek, "Complex Dynamics of a Discrete-Time Predator-Prey System with Ivlev Functional Response," Mathematical Problems in Engineering," Hindawi, vol 2018, Article ID 8635937, 2018, pp 1-15 https://doi.org/10.1155/2018/8635937

9. M. Moreno, F. Platania, "A cyclical square-root model for the term structure of interest rates,” Eur. J. Oper. Res., vol 241, 2015, pp 109-121.

10. A. George Maria Selvam, R. Janagaraj and D. Vignesh, "Allee effect and Holling type - II response in a discrete fractional order prey - predator model," IOP Conference Series: Journal of Physics, vol 1139 2018, pp 1-7.

11. A. George Maria Selvam, R. Janagaraj and Mary Jacintha, "Stability, bifurcation, chaos: discrete prey predator model with step size," International Journal of Innovative Technology and Exploring Engineering, vol 9, 2019, pp 3382-3387.

12. Saber Elaydi, "An Introduction to Difference Equations," Third Edition, Springer International Edition, First Indian Reprint, 2008.

13. J.D.Murray, "Mathematical Biology I: An Introduction," 3-e, Springer International Edition, 2004

14. Xiaoli Liu, Dongmei Xiao, "Complex dynamic behaviors of a discrete-time predator - prey system," Chaos, Solitons and Fractals, vol 32, 2007, pp 80 94.

15. Xinyu Zhu, Yunxian Dai, Qinglian Li and Kaihong Zhao, "Stability and Hopf bifurcation of a modified predator-prey model with a time delay and square root response function," Advances in Difference Equations vol 2017, 2017. DOI 10.1186/s13662-017-1292-1

16. B. D. Zheng, L.J. Liang and C.R. Zhang, "Extended Jurt criterion," Science China Math., vol 53, 2010, pp 1133-1150.

\section{AUTHORS PROFILE}

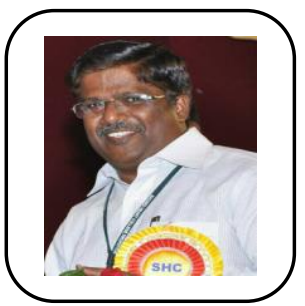

A. George Maria Selvam is an Associate Professor of Mathematics \& Head, Sacred Heart College, Tirupattur, Tamil Nadu, India. He holds a BSc and MSc from the University of Madras and $\mathrm{PhD}$ from Thiruvalluvar University. His research interests are Applied and Computational Mathematics, Mathematical Modelling, Nonlinear Analysis and Mathematical Biology.

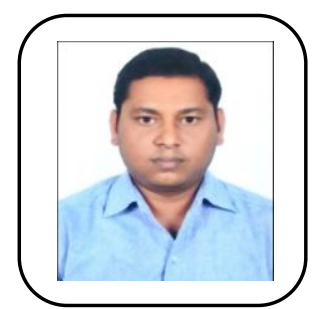

R. Janagaraj is a Research Scholar (FT) at Sacred Heart College, Tirupattur, Tamil Nadu, S.India and pursuing his PhD in Mathematics at Thiruvalluvar University, Tamil Nadu, India. He is a graduate in Mathematics from Bharadhidasan University, Tiruchirappalli, Tamil Nadu, India. His areas of specialization are Mathematical Modelling, Mathematical Biology and Dynamical System. 